\title{
Reconfiguration of 3 MV Marx Generator into a Modern High Efficiency System
}

\author{
Joni V. Klüss \\ Mississippi State University, USA
}

\author{
William Larzelere \\ Evergreen High Voltage, USA
}

\begin{abstract}
High voltage impulse generators are intended to last for long periods of time. Upon reaching the end of their technical lifetime, decisions concerning decommissioning, replacement, refurbishment or upgrading are needed. A new generator is a considerable investment. Significant reductions in material and labor costs can be made by repurposing still functional elements of the existing generator and redesigning the circuit for higher efficiency requiring fewer components. This paper describes the process of refurbishing the Mississippi State University (MSU) High Voltage Laboratory $3 \mathrm{MV}, 56 \mathrm{~kJ}$ impulse generator originally built in 1962 into a modern digital impulse generator system.
\end{abstract}

\section{Introduction}

In general, the basic function of an impulse voltage generator is the rapid application of capacitive energy to a load, i.e., the charging and discharging of a capacitor.

The impulse waveform can be approximated by a double exponential function,

$$
V(t)=V\left(e^{-t / \tau_{2}}-e^{-t / \tau_{1}}\right),
$$

shown in Fig. 1. Output voltage $\mathrm{V}(\mathrm{t})$ is the superposition of two exponential functions of different polarity [1]

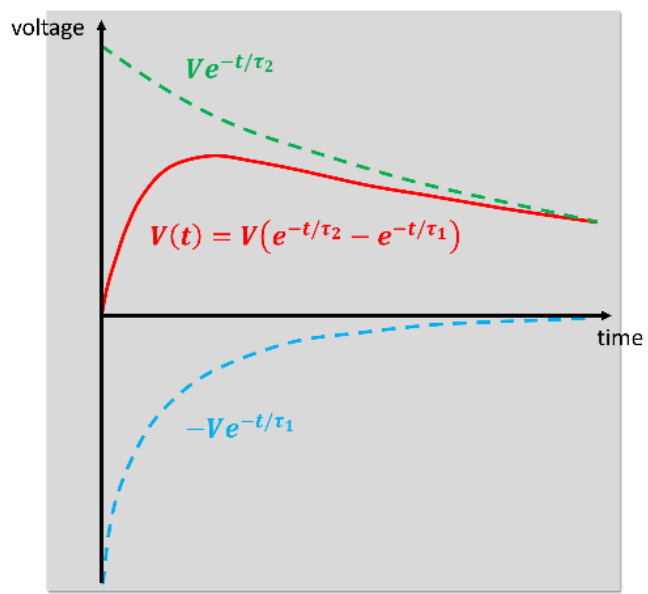

Fig. 1 - Double exponent waveform representing output voltage of an impulse generator.

This double exponential waveform can be produced by the circuit shown in Fig. 2 . In this circuit, capacitor $\mathrm{C}_{1}$ is charged by a source to voltage $V_{1}$ (resistor $R_{c}$ is a charging resistor between the source and the capacitor). The switch connecting $C_{1}$ to the rest of the circuit can either be triggered (trigatron - ignitable sphere gap), or self-ignited (e.g., increase voltage across open gap or decrease inter-electrode distance across gap to result in flashover). Upon closing of the switch, capacitance $\mathrm{C}_{2}$ is charged from $C_{1}$ through resistor $R_{1}$. At the same time, but slower ( since $\mathrm{R}_{2}>>\mathrm{R}_{1}$ ), both capacitors discharge through $R_{2}$. Output voltage $V_{2}$ appears across $C_{2}$.

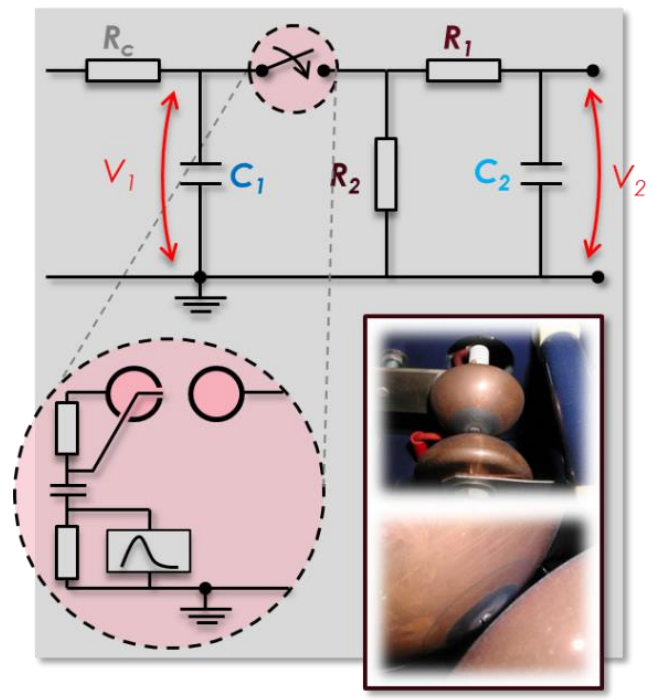

Fig. 2 - Single stage impulse generator circuit with a trigatron switch (triggered spark gap).

When the switch is closed (sphere gap is conducting), resistance $R_{2}$ can be momentarily neglected since $R_{2} \gg$ $\mathrm{R}_{1}$.

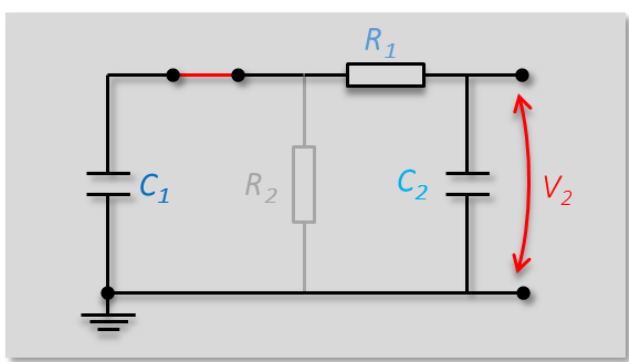

Fig. 3 - Charging of capacitor $\mathrm{C}_{2}$ upon ignition of switch.

Here, capacitors $\mathrm{C}_{1}$ and $\mathrm{C}_{2}$ are in series,

$$
C_{e q}=\frac{C_{1} C_{2}}{C_{1}+C_{2}}
$$

The time constant for this RC circuit in Fig. 3 is thus, 


$$
\tau_{1}=R_{1} \frac{C_{1} C_{2}}{C_{1}+C_{2}}
$$

After charging $\mathrm{C}_{2}$, both capacitors discharge though $\mathrm{R}_{2}$.

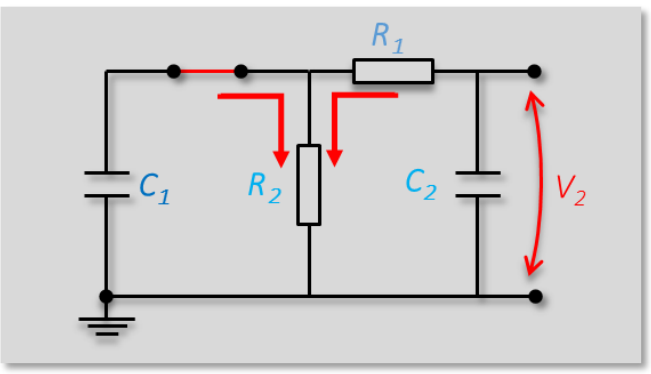

Fig. 4 - Discharging through resistor $\mathrm{R}_{2}$.

Now, $C_{1}$ and $C_{2}$ are in parallel, thus resulting in a time constant,

$$
\tau_{2}=R_{2}\left(C_{1}+C_{2}\right)
$$

These two time constants are used to form the double exponential curve in Fig. 1 where time constant $\tau_{1}$ defines the steepness of the waveform front, which is dependent on resistor $\mathrm{R}_{1}$. Time constant $\tau_{2}$ defines the decaying tail of the waveform and is related to resistor $\mathrm{R}_{2}$. Both parameters are also a function of capacitance $\mathrm{C}_{1}$ and $\mathrm{C}_{2}$,

Capacitor $\mathrm{C}_{1}$ is commonly referred to as the charging capacitor (a.k.a. discharge, surge, stage, or impulse capacitor) and this is considerable larger than capacitor $\mathrm{C}_{2}$ which is referred to as the load capacitance. This load capacitance can consist of any capacitive burden including the capacitance of the test object itself, the voltage divider, or any other additional capacitance added in parallel to the load for wave shaping purposes. Typically these capacitances are fixed and are not usually changed (permanent components of a system). In contrast, resistors $R_{1}$ and $R_{2}$ are components in the circuit that can be altered with little effort to achieve a desired output waveform.

IEC 60060-1 [2] defines the requirements for impulse waveforms. For standard lightning impulses (LI), front time $\mathrm{T}_{1}=1.2 \mu \mathrm{s} \pm 30 \%(0.84 \ldots 1.56 \mu \mathrm{s})$ and time to half-value $\mathrm{T}_{2}=50 \mu \mathrm{s} \pm 20 \%(40 \ldots 60 \mu \mathrm{s})$. For switching impulses (SI), time to peak $\mathrm{T}_{\mathrm{p}}=250 \mu \mathrm{s} \pm 20 \%$ $(200 \ldots 300 \mu \mathrm{s})$ and $\mathrm{T}_{2}=2500 \mu \mathrm{s} \pm 60 \%(1000 \ldots 4000$ $\mu \mathrm{s})$. Thus, to achieve such target values, one can modify the $R_{1}$ and $R_{2}$ resistors of the circuit.

The efficiency of the circuit can be evaluated by assessing the magnitude of the resultant double exponent waveform in Fig. 1 for purely capacitive loads and neglecting any circuit inductance. For the basic circuit in Fig. 2, output voltage $V_{2}$ is defined by the ratio of the capacitors [3],

$$
V_{2}=\frac{C_{1}}{C_{1}+C_{2}} V_{1}
$$

This provides the relationship for efficiency,

$$
\eta=\frac{V_{2}}{V_{1}}=\frac{C_{1}}{C_{1}+C_{2}}=\frac{1}{1+\frac{C_{2}}{C_{1}}}
$$

Efficiency thus increases as the ratio $\mathrm{C}_{2} / \mathrm{C}_{1}$ decreases. For this reason, charging capacitance $\mathrm{C}_{1}$ should always be much larger than load capacitance $C_{2}$. For $C_{1}>C_{2}$, the maximum stored energy is determined by $\mathrm{C}_{1}$,

$$
W=\frac{1}{2} C_{1} V_{1(\max )}^{2}
$$

A similar impulse circuit can also be used as shown in Fig. 5 where the placement of $R_{1}$ and $R_{2}$ has been shifted.

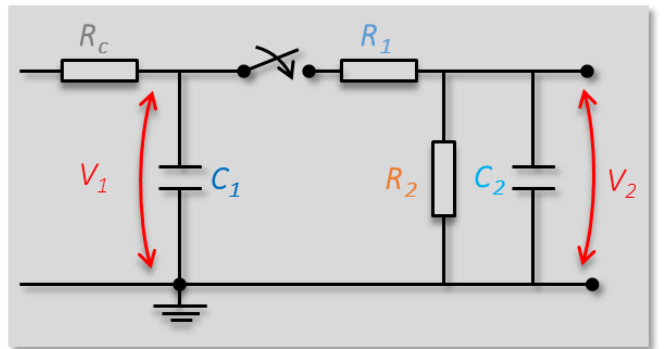

Fig. 5 - Alternative configuration of impulse circuit.

This circuit allows for the tail-shaping resistor $\mathrm{R}_{2}$ (a.k.a. earthing resistor) to be installed parallel to the test sample (load capacitance $\mathrm{C}_{2}$ ) - i.e., outside of the circuit. This has practical benefits such as easy access to the component to allow for fast modifications when striving to achieve standard waveform requirements. However, this configuration does require more space (clearance) and also has a negative influence on circuit voltage efficiency [4],

$$
\eta=\frac{C_{1}}{C_{1}+C_{2}} \frac{R_{2}}{R_{1}+R_{2}}=\frac{1}{1+\frac{C_{2}}{C_{1}}} \frac{1}{1+\frac{R_{1}}{R_{2}}}
$$

Here, the dependence on only $\mathrm{C}_{2} / \mathrm{C}_{1}$ is lost as the additional ratio $R_{1} / R_{2}$ is introduced. Since $R_{1}$ and $R_{2}$ influence the output waveform and can only be varied within the ranges needed to achieve standard LI and SI waveform parameters, the efficiency of the circuit in Fig. 5 is limited as shown in Fig. 6

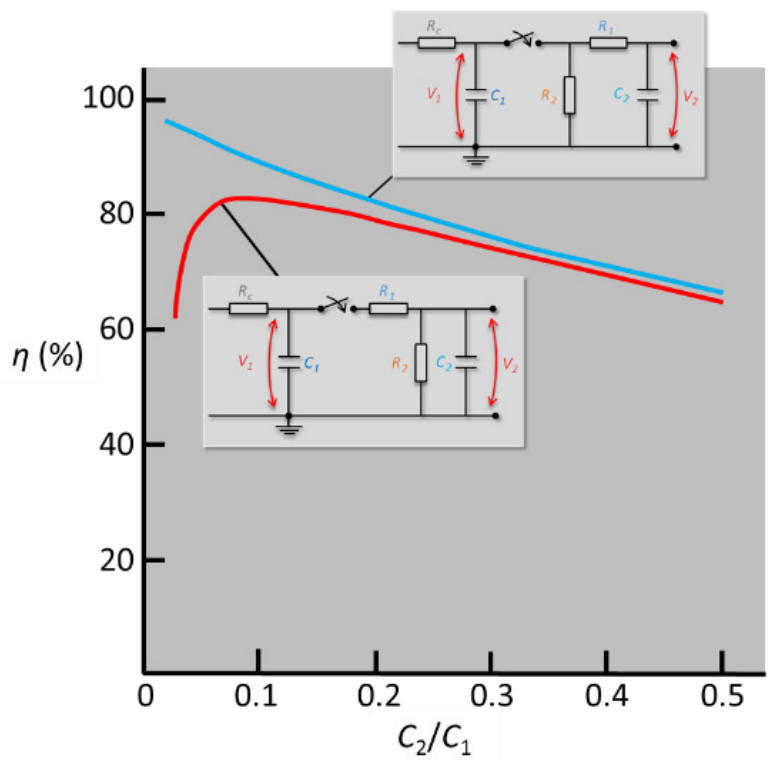

Fig. 6 - Efficiency $\eta=V_{2} / V_{1}$ as a function of ratio $C_{2} / C_{1}$ for standard lightning impulse (LI). Modified from [1]. 
The single stage circuits described (Fig. 2, Fig. 5) are commonly limited to under 200-300 kV. Reaching higher output voltages with such circuits poses several challenges such as $[1,5]$ :

- the increase of the physical size (and cost) of waveshaping circuit elements and the controlled ignition of large spacing spark gaps at very high voltages.

- difficulties in suppressing corona discharges during capacitor $\mathrm{C}_{1}$ charging.

\subsection{Multistage Generator}

A multistage impulse circuit, Marx generator (named after its inventor, Erwin Marx), utilizes the same principle as the single-stage generator, but cascades these circuits on top of each other (Fig. 7).

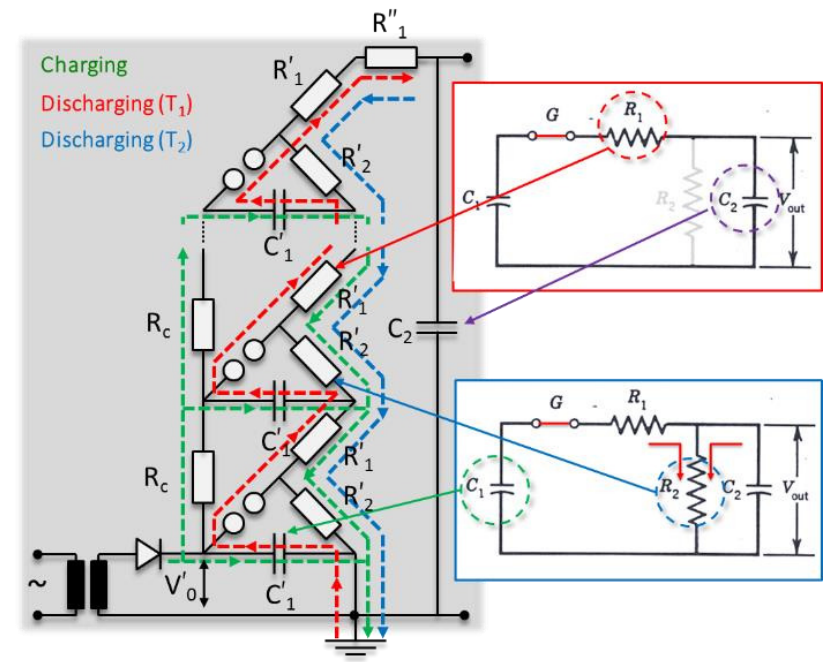

Fig. 7 - Multistage impulse generator circuit depicting the charging and discharging process.

The charging capacitors $C^{\prime}{ }_{1}$ are charged in parallel through the circuit resistors when the spark gaps are not conducting. Upon ignition, capacitors $C^{\prime}{ }_{1}$ are discharged in series. Similar to the single-stage circuit, resistance $\mathrm{R}_{1}^{\prime}$ shapes the front of the wave (front time $\mathrm{T}_{1}$ ) and $\mathrm{R}_{2}$ defines the tail (time to half-value $\mathrm{T}_{2}$ ). Capacitance $\mathrm{C}_{2}$ represents the capacitance of the test object itself together with any other capacitance in parallel with the generator (voltage divider, load capacitor, etc.).

Output voltage can be expressed as [6, 4],

$$
V_{2}(t)=\frac{V_{o}}{R_{1} C_{2}} \frac{\tau_{1} \tau_{2}}{\tau_{2}-\tau_{1}}\left(e^{-t / \tau_{2}}-e^{-t / \tau_{1}}\right)
$$

where time constants $\tau_{1}$ and $\tau_{2}$ are defined as in Eq. 3 and Eq. 4. The other relationships are,

$V_{0}=n V_{0}^{\prime} \quad(\mathrm{n}=$ number of stage $)$

$R_{1}=n R_{1}^{\prime}+R_{1}^{\prime \prime} \quad\left(R_{1}^{\prime \prime}=\right.$ external damping resistance $)$

$R_{2} \approx n R_{2}^{\prime}$

$C_{1}=\frac{C_{1}^{\prime}}{n}$

\subsection{Influential Factors}

The expressions for the presented time constants are valid for $C_{1}>C_{2}$. If load capacitance $C_{2}$ is large ( $>5$ $\mathrm{nF}$ ), it can become difficult to generate impulse waveforms within the specified standard tolerances especially when the inductance of the physical generator circuit is taken into account. Increased loading and higher inductance values result in overshoot in excess of the standards (IEC 60060-1 presents the maximum allowable relative overshoot magnitude as $10 \%$ [2]). Overshoot is an increase of impulse amplitude due to a damped oscillation at the impulse peak caused by the circuit inductance, load capacitance and front resistance.

Circuit inductance increases are difficult to avoid in large circuits (stray capacitance also increases with increasing physical dimensions). As the impulse generator circuit is large, each component and the circuit loop itself contributes some inductance. Inductance can vary from $10 \mu \mathrm{H}$ to several hundreds of $\mu \mathrm{H}$ - some multistage generators can be estimated as 3 $\mu \mathrm{H} /$ stage. Inductance of the external circuit (including test specimen) is approximated as roughly $1 \mu \mathrm{H} / \mathrm{m}$ [5].

Unless critical damping is applied with the proper value of front resistance, high inductance magnitudes may cause oscillations on the wave front which may persist past the peak value. When resistance $R_{1}$ is increased, wave front oscillations are damped, but the peak value of voltage is also reduced as is the front time. That is, steepness $(\mathrm{V} / \mathrm{s})$ is limited by circuit inductance. If the connection to the load from the generator is long, an additional external damping resistance $\left(\mathrm{R}_{1}{ }_{1}\right.$ in Fig. 7) helps damp oscillations caused by the inductance and capacitance of the external HV leads [1].

To remove oscillations for a given capacitance (fixed $\mathrm{C}_{1}$ and $\mathrm{C}_{2}$ ), the required damping resistance is defined by the inductance value [3],

$$
R_{1}=\sqrt{\frac{L\left(C_{1} / n+C_{2}\right)}{\left(C_{1} / n\right) C_{2}}}
$$

or if the circuit is viewed as an simple RCL circuit with $\mathrm{R}_{2}$ removed (due to its small influence on oscillations), critical resistance for the circuit to be non-oscillatory is generalized in [1] as,

$$
R_{1}=2 \sqrt{L / C}
$$

where

$$
C=\left(\frac{1}{C_{1}}+\frac{1}{C_{2}}\right)^{-1}=\frac{C_{1} C_{2}}{C_{1}+C_{2}}
$$

This generalization is rarely satisfied in practice - it restricts test sample capacitance to excessively small values (or extends $T_{2}$ to excessively long durations). Instead, replacing the factor of 2 with 1.18 produces overshoot within the acceptable $10 \%$ range [6]. Using this coefficient,

$$
R_{1}=1.18 \sqrt{L / C}
$$


and approximating front time as [7],

$$
T_{1} \approx 3 R_{1} C \quad \text { (for LI, } 1.2 / 50 \mu \mathrm{s} \text { ) }
$$

front resistance $R_{1}$ can be eliminated, giving,

$$
C \approx \frac{T_{1}^{2}}{12.5 L}
$$

If $\mathrm{T}_{1}=1.2 \mu \mathrm{s}$ and a typical value for inductance $\mathrm{L}=40$ $\mu \mathrm{H}$, the largest capacitance resulting in acceptable overshoot $(\leq 10 \%)$ is $\mathrm{C}=2.9 \mathrm{nF}$. If generator capacitance $\mathrm{C}_{1}=40 \mathrm{nF}$, then load capacitance $\mathrm{C}_{2} \leq 3.1$ $\mathrm{nF}$. At the maximum tolerance for $\mathrm{T}_{1}(=1.56 \mu \mathrm{s})$, this value for $\mathrm{C}_{2}$ is $\leq 5.5 \mathrm{nF}$. More information concerning the influence of internal inductance on the performance of an impulse generators is available in [8].

For inductive loads (e.g., reactor, transformer), while $\mathrm{T}_{1}$ may be attainable and efficiency is good, obtaining the proper $T_{2}$ is difficult. This can be improved by keeping effective resistance of the circuit large (connecting large $\mathrm{R}_{2}$ in parallel with test object), or increasing generator capacitance $\mathrm{C}_{1}$ by connecting stages in parallel $[4,5]$.

\section{Existing MSU Marx Generator Design}

The existing impulse generator at Mississippi State University's High Voltage Laboratory is a 1962 Nissin, type NIG, no. 1071, impulse generator (Fig 8, Fig. 9). With 20 stages and bipolar charging of $\pm 75 \mathrm{kV}(150 \mathrm{kV}$ per stage), theoretical output voltage is $3 \mathrm{MV}$. Stage capacitance of $0.25 \mu \mathrm{F}$ yields a total capacitance of 12.5 $\mathrm{nF}$ corresponding to an energy capacity of $56 \mathrm{~kJ}$. To maintain reasonable efficiency, maximum load capacitance is approximately 1-2 $\mathrm{nF}$ (normal series connection).

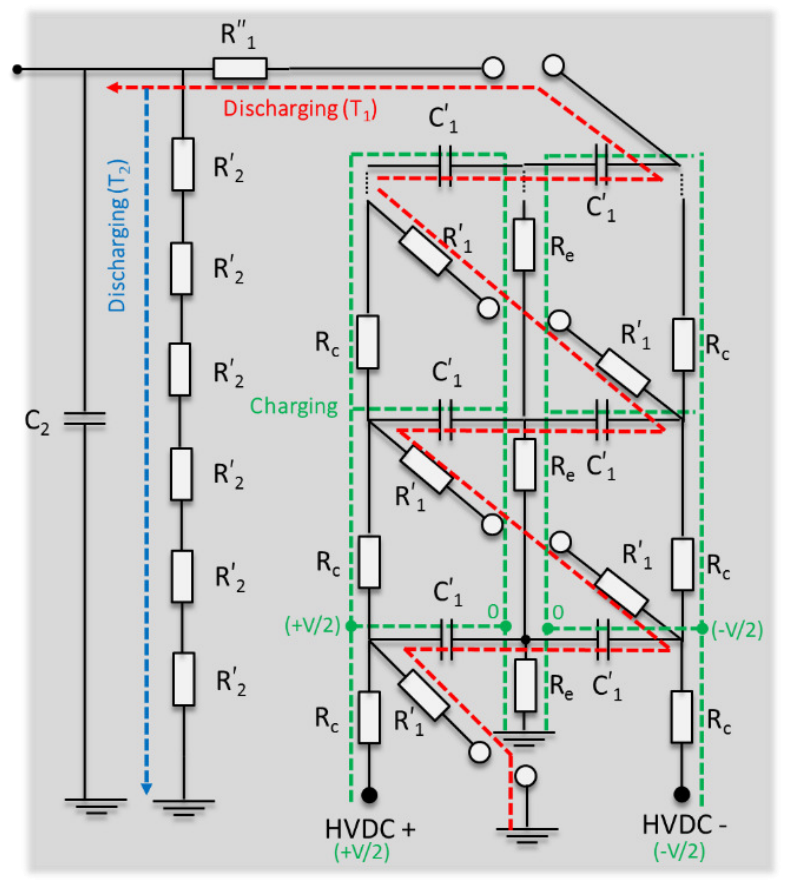

Fig. 8 - Schematic of MSU impulse generator showing bipolar charging and discharging process.

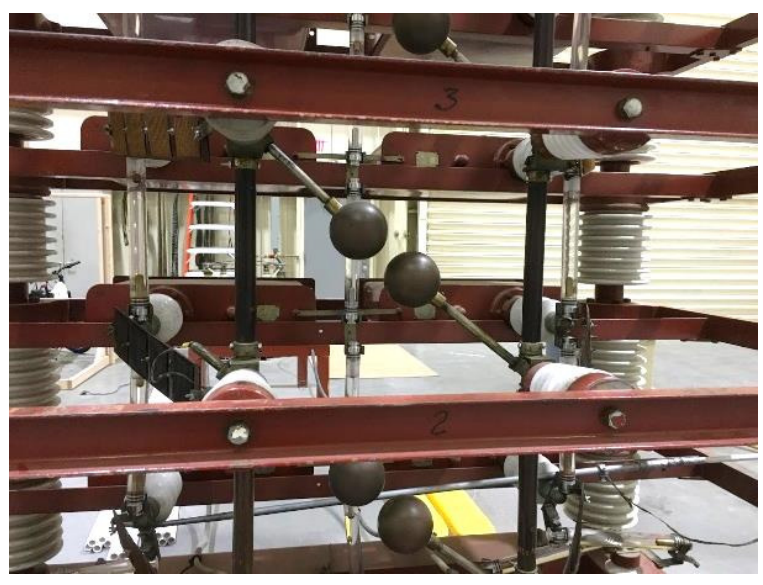

Fig. 9 - Components within a single stage of the MSU impulse generator.

Charging resistors $R_{c}$ and earthing resistors $R_{e}$ (for bipolar charging) are water resistors. They have good heat capacity for slow charging but are unstable due to changes in water properties (Fig. 10).

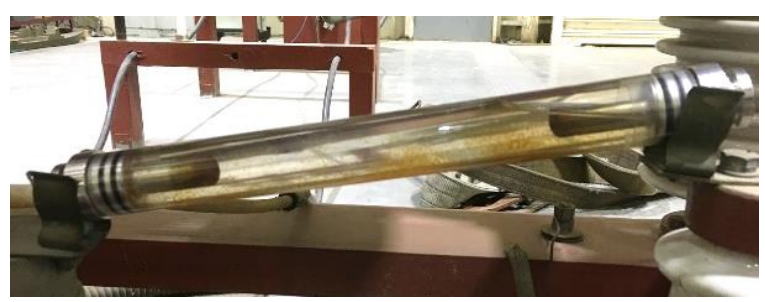

Fig. 10 - Charging process changes water properties within resistors over time as evident from the discoloration.

The wave-shaping resistors $\mathrm{R}_{1}$ and $\mathrm{R}_{2}$ are both wound (Nichrome) wire resistors (overlapping design for minimal inductance, Fig. 11). As the resistors age, they become prone to inter-turn short circuits as well as severed conductors. However, this design is easy to repair or replace with equivalent in-house constructed resistors.

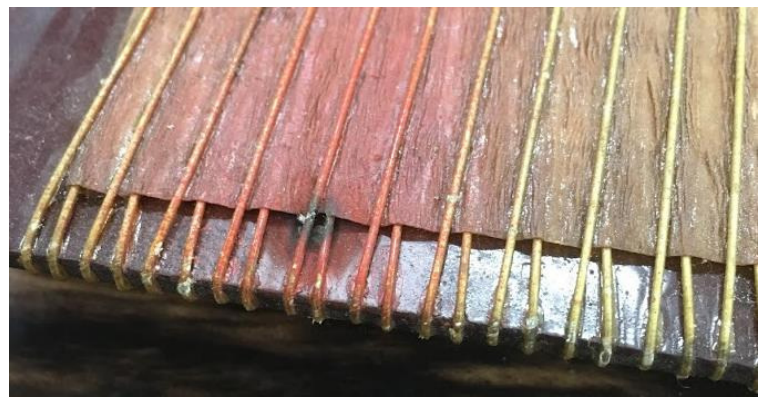

Fig. 11 - wire wound wave-shaping resistors with overlapping design.

The tail-shaping resistors $\mathrm{R}_{2}$ are all external (Fig. 12). This external design allows for easy access for modifying the waveform time parameters. Portions of the $R_{2}$ resistor can be tapped to increase front resistance $\mathrm{R}_{1}\left(\mathrm{R}_{1}{ }_{1}\right)$. Despite practical benefits, this design introduces larger current loops (inductance) and results in lower efficiency output. 


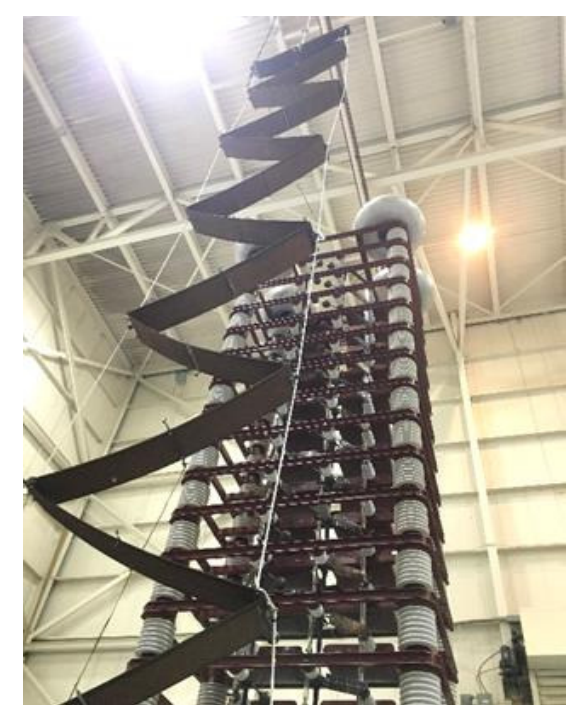

Fig. 12 - zigzag design of external tail resistors $R_{2}$.

The original design at one time had a trigger mechanism integrated into the bottom sphere gap. Over time different mechanism in the generator failed and were taken out. Currently, capacitors are charged to a predefined quantity whereupon the sphere are driven closer to cause self-ignition. Although functional, this form of repetitive displacement of sphere gaps continuously wears out the mechanical gears and components of the systems resulting in further repairs.

Although the capacitors appear to be in good condition, maximum charging voltage per capacitor has been restricted to $60 \mathrm{kV}(120 \mathrm{kV}$ per stage), limiting the theoretical output to $2.4 \mathrm{MV}$. At $75 \%$ efficiency, this allows for LI impulses up to $1.8 \mathrm{MV}$ for no-load test samples. Larger charging voltages are still possible, however, this increases the risk of damage to the wound wire resistors and inter-stage flashover across internal components in the generator. Flashover across the external $\mathrm{R}_{2}$ panels (bypassing portion of the resistance) also occurs at higher voltages if the zigzag construction is sufficient clearance is not maintained.

The generator circuit also lacks grounding for each stage. The existing circuit is only grounded at the supply which can leave considerable charge within the upper stage capacitors which are slowly drained through the large $R_{c}$ and $R_{e}$ unless an additional ground is applied. For this reason, in addition to grounding the generator, the test sample must also be grounded when working on the circuit.

Overall, the operation of the MSU impulse generator has been stripped down to the bear minimum functions still allowing for operation. Current operation is time consuming, inefficient, and unstable.

\section{New MSU Marx Generator Design}

Since the capacitors appear to be in good condition and sufficient for testing most apparatuses (with the exception of long cables and highly inductive loads), and the frame of the generator is also mechanically sound, it was decided that the circuit could be reconditioned with new components and controls.

This refurbishment includes:

- $\quad$ replacement of existing resistors

- replacement of gap system and drive

- reconfiguration of generator circuit

- $\quad$ adding new charging supply

- replacing control and measurement system

In the new generator circuit (shown in Fig. 13), the previously external tail resistance $\mathrm{R}_{2}$ are situated inside the generator structure similar to Fig. 7 and Fig 2. This configuration improves the efficiency of the system and also removes the need for bipolar charging. Compared to external tail resistance, access to internal components is more difficult. Nevertheless, the layout allows for easy addition and removal of parallel resistors to modify the front and tail times as needed. As only one charging resistor is needed per stage, this reduces the number of total components needed for the generator (resulting in reduced cost). The reduction in the number of internal components and the improved resistor design, together with the removal of bipolar charging, also reduces the risk of inter-stage flashover. The water resistors used for charging $\left(R_{c}\right)$ will be replaced with wound wire resistors enabling more stable charging as well as reducing the need for regular maintenance.

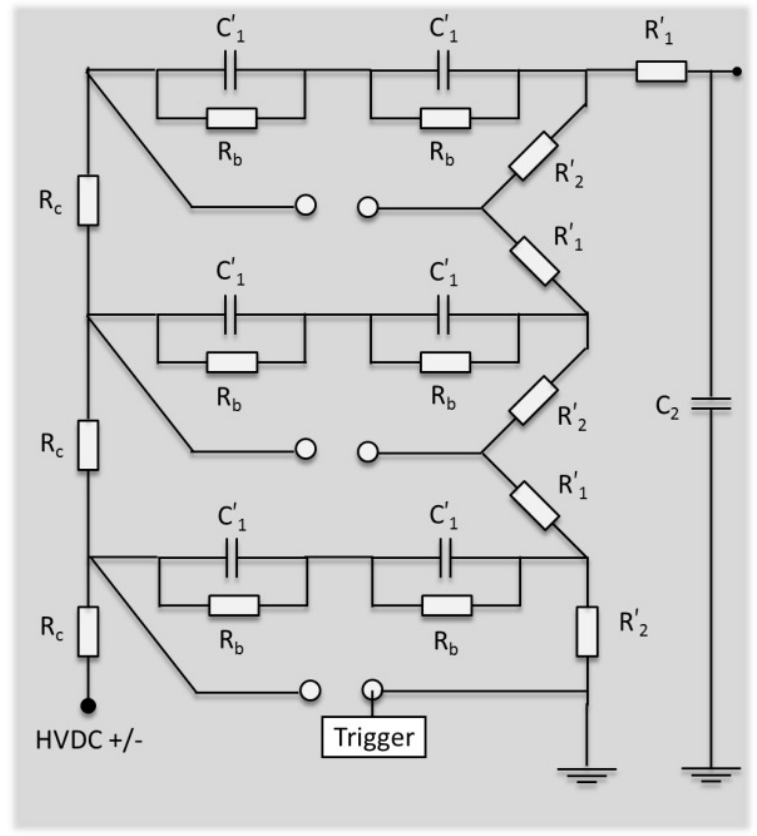

Fig. 13 - Schematic for new impulse generator circuit.

The sphere gap system will be completely replaced and fitted with a controlled trigger system (trigatron). The entire gap system will be within an enclosed column thus improving trigger performance.

A grounding belt system will be implemented to improve safety by ensuring discharge of all residual charges in each stage. 
To allow for adequate clearances for the gap system and drive components, the original bottom stage will be cleared. This will reduce the generator to 19 stages. However, the improvement in efficiency will compensate for the one sacrificed stage. This also results in two spare replacement capacitors in case of failures. Reutilizing the original capacitors is a major reduction in costs - the generator capacitance (energy) is the major expense for impulse generators.

Another significant expense is the modernization of the measurement and control system. As mentioned earlier, the functionality of the original design has been reduced to its bear minimum still allowing for successful operation. Capacitors are charged with large gap spacings and self-ignition is initiated by driving the sphere gaps closer. Output voltage is recorded by a digitizer via a damped capacitive divider. The new systems will have integrated functions for controlling the generator, capturing the data, and performing postprocessing according to modern standard requirements. This single unit with an industrialized computer and optical interface is also significantly smaller than the original control configuration, thereby freeing up considerable space in the control room.

Naturally, output efficiency is dependent on the test load but the intrinsic efficiency of the reconfigured circuit can be approximated as exceeding 93\%. In general, to maintain efficiency above $80 \%$, it is suggested that capacitive loads should not exceed $15 \%$ of the net series capacitance of the number of stages. For the full 20-stage generator with series capacitance C $=12.5 \mathrm{nF}$, this would restrict loads to under $2 \mathrm{nF}$ which is satisfactory for the expected test loads at MSU. Since, the original capacitors will be kept, this value does not change in the new design. It should be noted that since each stage is a complete impulse generator, reduced number of stages can easily be used to test higher load capacitances at lower voltages. Stage paralleling for much higher load capacitances is also possible. For the new design, the target internal inductance for each stage wiring is in the range of 1-2 $\mu \mathrm{H}$ plus the inductance of the capacitors and front resistors. This will be verified upon completion of the renovation.

\section{Progress}

The complete refurbishment process and design details have been finalized and the project is expected to start in the summer of 2017. Originally, the rebuild was designed to be implemented in stages. This is a suitable approach for limited budgets. The first initial investment would be used to modernize the measurement and control system. This could be installed to operate with the existing generator and supply. Upon procurement of further funds, the next stage could be employed replacement of generator charging supply. Next, with a renovated controls and supply scheme, the impulse generator itself could be updated. This can also be done in stages. Half of the generator stages could be bypassed and fitted with new components while the other half remains operations. In this manner, output capacity is restricted but service interruption is minimal operations of the impulse generator is maintained throughout the entire refurbishment process. As mentioned earlier, this is suitable for limited budget, however, the total refurbishment time is extended. This allows for more time to obtain further funding but also exposes the project to the risk of never being completed.

To minimize the renovation time, once the process is started, all of the presented upgrades will be implemented at once for the impulse generator system at MSU.

\section{Conclusion}

Ideally, a full replacement with a completely new system and generator would be optimal as compatibility and integration issues are avoided. However, the cost of a new generator is significant, and is not always an option. If the original capacitors (which are the main expense) in an existing system are still in good condition, a customized refurbishment may be feasible. The entire system must be assessed as a whole to determine potential savings. The presented renovation of the MSU impulse generator is not cheap, but it is still below the price of a completely new system, making this a worthwhile investment.

\section{References}

[1] E. Kuffel, W.S. Zaengle, and J. Kuffel, High Voltage Engineering Fundamentals, $2^{\text {nd }}$ Ed., Newnes, Oxford, 2000 pg. 55-64]

[2] IEC 60060-1, High-voltage test techniques - Part 1: General definitions and test requirements, 2010.

[3] H.M. Ryan, High Voltage Engineering and Testing, $2^{\text {nd }}$ Ed, IEE, London, 2001, pg. 519-522

[4] D. Kind, and K. Feser, High-Voltage Test Techniques, $2^{\text {nd }}$ Ed., Vieweg/SBA Publications, New Delhi, 1999, pg. 47-54.

[5] M.S. Naidu, and V. Kamaraju, High Voltage Engineering, $2^{\text {nd }}$ Ed., McGraw-Hill, New York, 1996, pg. 136.

[6] M. Aro, J. Elovaara, M. Karttunen, K. Nousiainen, and V. Palva, Suurjännitetekniikka, $4^{\text {th }}$ Ed., Otatieto, Helsinki, 2015, pg. 388-391.

[7] Beyer M, Boeck W, Möller K, and Zaengal W, Hochspannungstechnik, Springer Verlag, 1992

[8] W. Larzelere, J. Hällström, A-P. Elg, A. Bergmann, J. Klüss, Y. Li, and L. Zhou, Measurement of the Internal Inductance of Impulse Voltage Generators and the Limits of LI Front Times, 20 ${ }^{\text {th }}$ International Symposium on High Voltage Engineering, Buenos Aires, Argentina, Aug 27 - Sept 1, 2017. 